\title{
Zur routinemäßigen Bestimmung von Hydroxyprolin im Harn
}

\author{
Von H. HAURY' ${ }^{1}$ ) \\ Aus den wissenschaftlichen Laboratorien der Firma Dr. H. Haury, Mïnchen
}

(Eingegangen am 19. April 1971)

Es wird eine für den Routinegebrauch im klinisch-chemischen Laboratorium geeigncte Methode zur Bestimmung von Hydroxyprolin im Harn beschrieben. Durch Vergleich mit anderen Methoden, Berechnung des Variationskoeffizienten und Wiederfindungsversuche ist die Brauchbarkeit erwiesen.

\section{Routine determination of urinary bydroxyproline}

A routine method is described, which is suitable for the determination of urinary hydroxyproline in the clinical chemical laboratory. The suitability of the method is shown by comparison with other methods, calculation of the variation coefficients and recovery experiments.

Die Hydroxyprolin-Ausscheidung ist ein $\mathrm{MaB}$ für den Kollagenstoffwechsel und daher vor allem bei Knochenerkrankungen diagnostisch wichtig. Freies Hydroxyprolin wird nur in Spuren ausgeschieden. Nur bei der sehr seltenen familiären Hydroxyprolinämie ist die Ausscheidung erhöht. Die Exkretion des peptidartig gebundenen Hydroxyprolin ist erhöht bei hochgradiger Osteoporose, Osteogenesis imperfecta, Osteodystrophia deformans (PAGET), MARFAN-Syndrom, Knochen-Tuberkulose und neoplastischen Knochenmetastasen (siehe z. B. 1).

Bei einer Hyperthyreose ist der HydroxyprolinSpiegel im Urin erhöht $(2,3)$. Die zitierten Autoren fanden eine enge Korrelation zwischen dem in der Hydroxyprolin-Ausscheidung dokumentierten Kollagenabbau und der Höhe des proteingebundenen Jods. Umgekehrt sind bei Hypothyreose verminderte Werte festgestellt worden (4). Auch beim Hyperparathyreoidismus werden meist erhöhte Hydroxyprolin-Werte festgestellt (z. B. 5).

Bei erhöhter Aktivität der alkalischen Phosphatase erlaubt die Bestimmung der Hydroxyprolin-Ausscheidung die Unterscheidung zwischen Knochenerkrankungen (Hydroxyprolin im Harn erhöht) und Lebererkrankungen (Hydroxyprolin normal) (6). Bei der primär-chronischen Polyarthritis findet man bei den meisten Patienten eine erhöhte Hydroxyprolin-Ausscheidung schon im ersten Stadium, in dem noch keine Gelenkveränderungen erkennbar sind, keine Funktionseinschränkung vorhanden ist und nur Anlaufschmerz besteht. In diesem Stadium sind andere Untersuchungen oft noch negativ. Dagegen normalisiert sich die Hydroxyprolin-Ausscheidung im fortgeschrittenen Stadium der primär-chronischen Polyarthritis (7). Bei Kindern ist die Hydroxyprolin-Ausscheidung während des Wachstums (insbesondere Knaben 12-16 Jahre, Mädchen 11-14 Jahre) stark erhöht (8).

\footnotetext{
1) Unter technischer Mitarbeit von Frl. B. Sengling.
}

Wie diese kurze Ubersicht gezeigt hat, ist der diagnostische Wert der Hydroxyprolin-Bestimmung beträchtlich. Wenn sie bisher noch keine weitere Verbreitung gefunden hat, so liegt das an den komplizierten Methoden, die trotz einer kaum überschaubaren Zahl von Modifikationen bisher nur unwesentlich an Brauchbarkeit für den Routinegebrauch gewonnen haben.

Hydroxyprolin liegt im Urin in Form von Di- und Tripeptiden vor, insbesondere als Prolylhydroxyprolin (9). Neuerdings sind auch Hydroxyprolin enthaltende Glycopeptide und Polypeptide in menschlichem Harn gefunden worden (10). Der Bestimmung muß daher eine saure (11-17) oder alkalische $(14,18)$ Hydrolyse vorausgehen. Während in der Lebensmittelchemie mitunter Schwefelsäure verwendet wird (19), ist in der klinischen Chemie allgemein Salzsäure gebräuchlich. Das freie Hydroxyprolin wird zu einer Verbindung bisher noch unbekannter Struktur oxydiert (die früher von mehreren Autoren geäußerte Annahme, es handele sich bei dem Oxydationsprodukt um 2-Pyrrol-Karbonsäure oder um Pyrrol $(20,21)$, ist von BERGMAN und LOXLEY (11) widerlegt worden). Das Oxydationsprodukt bildet mit $p$-Dimethylaminobenzaldehyd einen Farbstoff, dessen Intensität der Hydroxyprolin-Konzentration proportional ist. Früher wurde als Oxydationsmittel nach Neuman und Logan (22) Wasserstoffperoxid verwendet. Eine wesentliche Verbesserung erfolgte mit der Einführung von Chloramin $T$ durch H. StEgEmanN (21), auf dessen verdienstvolle grundlegende Veröffentlichung die heute verwendeten Modifikationen der Hydroxyprolin-Bestimmung zurückzuführen sind.

Die Oxydation mit Wasserstoffperoxid hat den Nachteil, $\mathrm{da} \beta$ Úberschüsse dieses Oxydationsmittels nicht leicht zu entfernen sind. Durch die von Stegemann eingeführte Perchlorsäure witd nach Bildung des Oxydationsproduktes überschüssiges Chloramin $T$ zer- 
stört und gleichzeitig das saure Medium für die Reaktion mit $p$-Dimethylaminobenzaldehyd vorbereitet. Prockop und UdENFrIEND $(15,20)$ verwenden zur Zerstörung des überschüssigen Chloramin T Natriumthiosulfat und anschließend zum Ansäuern Schwefelsäure. Perchlorsäure hat demgegenüber den Vorteil, $\mathrm{da} ß$ nur ein Reagenz zugesetzt werden muß, das beide Funktionen erfüllt. Gegenüber der Verwendung von Schwefelsäure bietet Perchlorsäure den Vorteil, die Methode auch dann anwenden zu können, wenn Magnesium-, Calcium- oder Barium-Ionen vorhanden sind.

Viele Autoren führen die Hydrolyse bei Temperaturen von mehr als $100^{\circ}$ durch, die im klinischen Laboratorium schwer $\mathrm{zu}$ handhaben und $\mathrm{zu}$ kontrollieren sind. Vielfach ist vorgeschlagen worden, nach der Hydrolyse zur Entfernung der Salzsäure zur Trockne einzudampfen. Dazu sind Temperaturen um $140^{\circ}$ notwendig, die für den Routinegebrauch vermieden werden müssen. Nach unseren Untersuchungen genügen $100^{\circ}$ für die Hydrolyse. Die Erhöhung der Temperatur auf $105^{\circ}$ nach BERGMAN und LOXLEY $(23,12)$ oder $107^{\circ}$ (17) bringt keine höhere Ausbeute, ebenso wenig wie Erhöhung auf $124^{\circ}(20), 125^{\circ}$ (14) oder noch höher $\left(140^{\circ}\right)$. Die Entfernung der Salzsäure erfolgt am besten durch Neutralisation, wobei Lithiumhydroxid wegen der guten Löslichkeit und geringster Störung des weiteren Analysenganges anderen Alkalien gegenüber vorzuziehen ist (12).

Für die weitere Behandlung des Hydrolysates sind zur Verbesserung der Spezifität enzymatische (24), chromatographische $(14,20,25,26)$ und Extraktions- (20), bzw. Destillationstechniken (27) vorgeschlagen worden. Sie sind in der Praxis entweder kaum durchführbar (enzymatisches Verfahren) oder umständlich und unnötig. Die chromatographische Entfernung störender Substanzen führt zu Verlusten an Hydroxyprolin (13). Die von PRockop und UDENFrIEND (23) vorgeschlagene Extraktion mit Toluol hat eine geringere Reproduzierbarkeit zur Folge (13).

Eine Routine-Methode soll zwar möglichst einfach sein. Auf strenge Anforderungen in bezug auf Spezifität, Reproduzierbarkeit und Vollständigkeit darf aber nicht verzichtet werden. Der Kreis der zahlreichen Möglichkeiten zur Hydroxyprolin-Bestimmung ist nach dem oben Erwähnten schon stark eingeengt. Um die beste Methode zu finden, konnten wir uns daher auf eine nähere Untersuchung der Arbeiten von STEGEMANN (21) in der Modifikation von StegEMANN und Stalder (17), von Firschein und ShiLI (14), von Ellis und Goldberg (13) und von Bergman und LOXLEY $(11,12,23)$ beschränken.

Wir fanden bei der Methode nach FirsChein und SHILl (14) einen Variationskoeffizienten von $27 \%$, bei der Methode von Stegemann und Stalder (17) von $7,1 \%$ und bei der Methode von Bergman und LoxLEY (12) in unserer Modifikation von 3,4\% (siehe Tab. 1). ELIIs und Goldberg (13) berechneten für die von ihnen
Tab. 1

Variationskoeffizienten. Für jede Methode wurden 16 Hydroxyprolin-Bestimmungen in jeweils der gleichen Urinprobe durchgeführt

\begin{tabular}{|c|c|c|c|}
\hline & \multicolumn{3}{|c|}{$\begin{array}{l}\text { Ergebnisse der Hydroxyprolin-Bestimmung } \\
\text { im Harn nach drei Methoden }\end{array}$} \\
\hline & $\begin{array}{l}\text { FIRSCHEIN u. } \\
\text { SHILL (14) }\end{array}$ & $\begin{array}{c}\text { STEGEMANN } \\
\text { u. STALDER } \\
\text { (17) }\end{array}$ & $\begin{array}{l}\text { BERGMAN u. } \\
\text { LOXLEY (12). }\end{array}$ \\
\hline$\overline{\mathbf{x}}(\mathrm{mg} / \mathrm{l})$ & 72,4 & 77,5 & 24,2 \\
\hline s & 19,5 & 5,5 & 0,83 \\
\hline $\begin{array}{l}\text { Variationskoeffi- } \\
\text { zient \% } \\
\text { Variationsbreite }\end{array}$ & 27 & 7,1 & 3,4 \\
\hline $\bar{x} \pm s$ & $61,5-94,5$ & $67,5-85,7$ & $23,0-26,0$ \\
\hline
\end{tabular}

verglichenen Methoden folgende Variationskoeffizienten: 17,8 mit der Methode von Prockop und UdENFRIEND (20), 2,0 mit der von StegemanN und Stalder (17) und 1,7 mit der von Bergman und LoXley (12), in die sie die Verwendung sogenannter „innerer Standards" eingeführt haben.

Auch die Ergebnisse unserer Versuche zur Wiederfindung den Urinproben zugesetzten Hydroxyprolins stimmen mit denen von Ellis und Goldberg (13) im Prinzip überein (Tab. 2). Mehrere weitere von uns untersuchte Methoden erbrachten wesentlich schlechtere Ergebriisse, schon allein wegen ihrer geringeren Präzision.

Tab. 2

Ubersicht über die wichtigsten Wiederfindungsversuche. Die Zusätze erfolgten bei ELLIS und GoLDBERG anscheinend nach, bei den eigenen Versuchen vor der Hydrolyse

\begin{tabular}{|c|c|c|c|c|}
\hline & \multicolumn{2}{|c|}{$\begin{array}{l}\text { Wiederfindungs- } \\
\text { versuche von ELLIS } \\
\text { und GoLDBERG (13) } \\
(23 \text { Urinproben) }\end{array}$} & \multicolumn{2}{|c|}{$\begin{array}{c}\text { Eigene } \\
\text { Wiederfindungsversuche } \\
\text { (20 Urinproben) }\end{array}$} \\
\hline & $\begin{array}{l}\text { STEGEMANN } \\
\text { u. STALDER } \\
(17)\end{array}$ & $\begin{array}{l}\text { BERGMAN } \\
\text { u. LOXLEY } \\
(12)\end{array}$ & $\begin{array}{l}\text { STEGEMANN } \\
\text { u. STALDER } \\
(17)\end{array}$ & $\begin{array}{l}\text { BERGMAN } \\
\text { u. LOXLEY, } \\
\text { modifiziert }\end{array}$ \\
\hline $\bar{x}(\%)$ & 77,4 & 87,0 & 81 & 95 \\
\hline$\pm \mathrm{s}(\%)$ & 13,8 & 8,6 & 19,2 & 15,0 \\
\hline Extremwerte (\%) & $44-100$ & $63-100$ & $72-98$ & $81-112$ \\
\hline
\end{tabular}

Durch die Adsorption an Ionenaustauscher sind Hydroxyprolin-Verluste offensichtlich unvermeidbar. STALDER (26) berichtet, daß den Harnproben zugesetztes Hydroxyprolin nur zu $80 \%$ wiedergefunden wurde. Auch Meilman und Mitarbeiter (9) berichten, $\mathrm{da} ß$ durch Vorbehandlung mit einem Ionenaustauscher $10 \%$ Hydroxyprolin verloren gehen. Die Mitteilung von Strgemann und Stalder (17), nach der an Ratten verfüttertès Hydroxyprolin mit $\pm 3 \%$ im Urin wiedergefunden wurde, konnte mit unsererVersuchsanordnung bei menschlichem Urin nicht bestätigt werden.

Nach den geschilderten Versuchsergebnissen ist verständlich, daß wir die Methode von Bergman und LOXIEY (12) als Verfahren der Wahl empfehlen. In Übereinstimmung mit ElLIS und GoldBerg (13) wurde die Hydrolysentemperatur von $105^{\circ}$ auf $100^{\circ}$ herabgesetzt und der sogenannte „innere Standard“ verwendet. Dabei führt man zu jeder Bestimmung einen in Isopropanol gelösten Standard mit, dem Hydrolysat zugesetzt wurde. Dadurch wird nicht nur jede Störung durch die Eigenfarbe des Urins, sondern auch der Ein- 
fluß ursprünglich vorhandener oder während der Hydrolyse gebildeter unspezifischer Chromogene ausgeschaltet.

Unsere Versuche zeigten, daß die Dauer der Hydrolyse 7-8 Stunden betragen sollte. Verkürzte Zeiten führen $\mathrm{zu}$ unvollständiger Hydrolyse, längere $\mathrm{zu}$ keiner höheren Ausbeute. Die Eichkurve ist innerhalb des in Betracht kommenden Extinktionsbereiches (Photometer Eppendorf, $546 \mathrm{~nm}, 1 \mathrm{~cm}$-Küvette) bis zu einer Extinktion von 1,93 (entsprechend $3 \mathrm{~g} / 1$ Hydroxyprolin) gerade (Abb. 1).

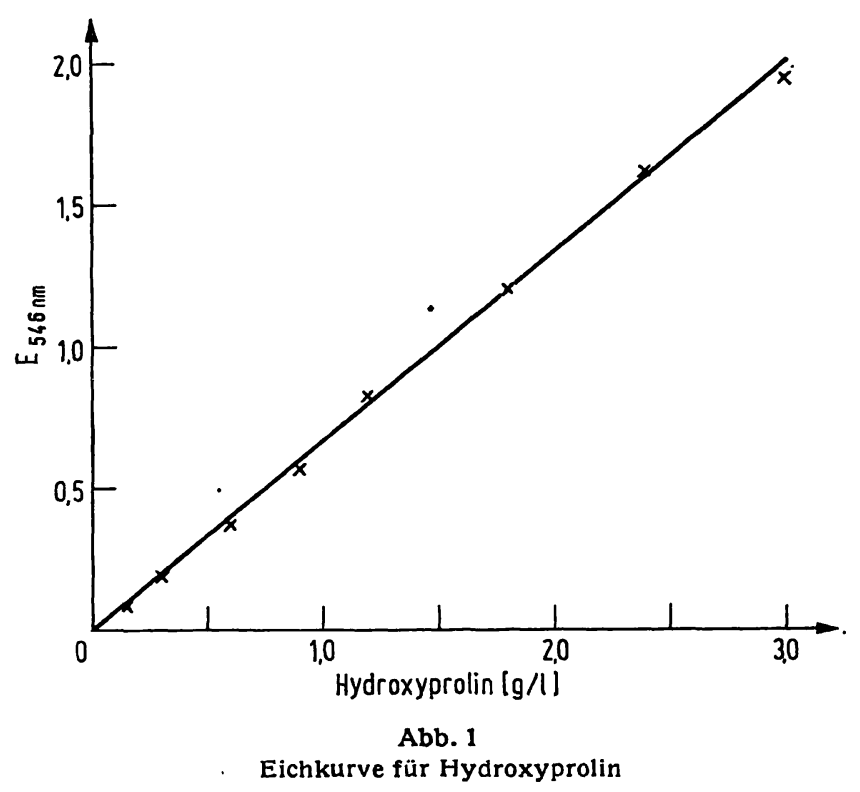

Außerdem wichen wir noch in folgenden eingehend exprobten Punkten von der Methode von Bergman und LOXLEY (12) bzw. Eliss und Goldberg (13) ab:

1. Statt von $5 \mathrm{ml}$ Urin wurde von $1 \mathrm{ml}$ ausgegangen.

2. Für die Hydrolyse wurden $9,5 \mathrm{~cm}$ hohe Zentrifugengläser mit Schliffstopfen aus Glas verwendet. Sie wurden in einem Aluminiumblock oder Trockenschrank auf $100^{\circ}$ erhitzt. Abspringen der Schliffstopfen beobachteten wir nur, wenn die Gläser kalt in einen heißen Wärmeblock gestellt wurden. Dagegen kann der Trockenschrank vorgewärmt sein.

3. Bei Zugabe von exakt 7N Salzsäure zum Urin läßt sich die Neutralisation des Hydrolysats mit genau eingestellter Lithiumhydroxidlösung so genau durchführen, $\mathrm{da} B$ auf eine Einstellung des pH-Wertes unter Zuhilfenahme eines Indikators verzichtet werden kann.

4. Die Farbentwicklung ist nach $20 \mathrm{Min}$. im Wasserbad von $60^{\circ}$ beendet.

5. Es brauchen nicht 2, sondern nur 1 Standard mitgeführt zu werden.

6. Das Extinktionsmaximum liegt bei etwa $570 \mathrm{~nm}$. Bergman und Loxley (12) messen bei 562, Ellis und Goldberg (13) bei $560 \mathrm{~nm}$. Zwischen 540 und 580 abgelesene Werte sind nach unseren Versuchen brauchbar.
7. Uber die Berechnung machen BERGMAN und LOXLEY (12) keine Angaben. Bei Ellis und Goldberg (13) wird der Hydroxyprolin-Gehalt in $1 \mathrm{ml}$ Hydrolysat aus einer relativ komplizierten Graphik entnommen. Wir ermitteln den Hydroxyprolin-Gehalt im Urin selbst durch Berechnung.

8. Die Lösung von $p$-Dimethylaminobenzaldehyd in Perchlorsäure wird von BERGMAN und LOXLEY (11) $z$ war jeweils frisch angesetzt, soll aber einige Wochen haltbar sein. Nach unseren Erfahrungen ist sie jedoch nur 2-3 Tage haltbar. Ähnliche Erfahrungen haben ARNETH und HAMM gemacht (28), die es als empfehlenswert bezeichnen, das Aldehyd-Perchlorsäure-Reagens möglichst immer frisch anzusetzen.

9. $\mathrm{Zu}$ dem verwendeten Puffer ist zu sagen, daß frühere Bearbeiter häufig keine Angaben über den $\mathrm{pH}$-Wert machen. Strgemann (21) gibt an etwa 6 , Bergiran und LOXLEY (11) sowie WoessNer (29) genau 6 , dagegen KIvirikko und Mitarbeiter (15) 8,7. Uns bewährte sich ein $\mathrm{pH}$-Wert von 6,3 .

\section{Methodik}

Es wird vorgeschlagen, die Hydroxyprolinbestimmung wie folgt durchzuführen:

\section{Gerãte}

Zentrifuge

Zentrifugengläser mit Schliffstopfen

Wärmeblock bis $100^{\circ}$

Wasserbad bis $60^{\circ}$

Meßzylinder $5 \mathrm{ml}$ oder $10 \mathrm{ml}$

Filter- oder Spektralphotometer

Meßbereich $540-580 \mathrm{~nm}$, Spektralphotometer $570 \mathrm{~nm}$.

\section{Reagenzien ${ }^{2}$ )}

1. Standard $5 \mathrm{mg} / 1$ Hydroxyprolin in Isopropanol wasserfrei.

2. Pufferlösung. $37 \mathrm{~g}$ Natriumacetat krist., $45,38 \mathrm{~g}$ Tri-Natriumcitrat-5,5-Hydrat und 5,5 g Citronensäure krist. werden in $385 \mathrm{ml}$ Isopropanol gelöst und mit destilliertem Wasser auf 11 aufgefüllt. 3. Chloramin T. $70 \mathrm{mg}$ Chloramin $\mathrm{T}$ werden in $1 \mathrm{ml}$ Wasser gelöst und auf $5 \mathrm{ml}$ mit Puffer aufgefüllt. Die Lösung ist höchstens 2 Stunden haltbar.

4. EhrLICHs Reagenz. $0,7 \mathrm{~g} p$-Dimethylaminobenzaldehyd werden in $1,6 \mathrm{ml} 60$ proz. Perchlorsäure gelöst und auf $4 \mathrm{ml}$ mit wasserfreiem Isopropanol aufgefüllt. Die Lösung ist $2-3$ Tage haltbar.

5. 60proz. Perchlorsäure.

6. 4,8proz. Lithiumhydroxid-Lösung.

7. 7 Salzsäure.

8. Wasserfreier Isopropylalkohol.

\section{Durchführung}

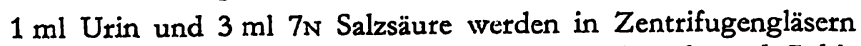
mit Schliff und Schliffstopfen angesetzt, geschüttelt und 7 bis $8 \mathrm{Stdn}$. bei $100^{\circ}$ im Wärmeblock oder Wärmeschrank stehen gelassen. Nach Abkühlung des Hydrolysats wird das Volumen gemessen und, wenn nötig, mit $7 \mathrm{~N}$ Salzsäure auf $4 \mathrm{ml}$ aufgefüllt.

Anschließend wird $1 \mathrm{ml}$ Hydrolysat entnommen und mit $3 \mathrm{ml}$ 4,8 proz. Lithiumhydroxid-Lösung und $0,1 \mathrm{ml} 7 \mathrm{~N}$ Salzsäure versetzt. Aus diesem neutralisierten Hydrolysat werden $3 \mathrm{mal} \mathrm{je}$ $0,5 \mathrm{ml}$ in Reagensgläser pipettiert und wie folgt weiterbehandelt:

2) Enthalten in Haurytest „Hydroxyprolin“ (außer Salzsäure und Isopropanol). 


\begin{tabular}{lcccc}
\hline & Analyse & $\begin{array}{c}\text { Analysen- } \\
\text { leerwert }\end{array}$ & Standard & $\begin{array}{c}\text { Reagenzien- } \\
\text { leerwert }\end{array}$ \\
\hline $\begin{array}{l}\text { Hydrolysat } \\
\text { Destilliertes }\end{array}$ & 0,5 & 0,5 & 0,5 & - \\
$\begin{array}{l}\text { Wasser } \\
\text { Isopropanol }\end{array}$ & - & - & - & 0,5 \\
wasserfrei & 1,0 & $-1,0$ & - & 1,0 \\
$\begin{array}{l}\text { Standard } \\
\text { Chloramin } \\
\text { T-Lösung }\end{array}$ & 0,5 & - & 0,5 & 0,5
\end{tabular}

sehr gut mischen und nach 4 Minuten zugeben:

\begin{tabular}{|c|c|c|c|}
\hline $\begin{array}{l}\text { EHRLICHS } \\
\text { Reagenz } \\
\text { Chloramin }\end{array}$ & 1,0 & 1,0 & \\
\hline $\mathrm{T}$-Lösung & - & 0,5 & - \\
\hline
\end{tabular}

Gläser $20 \mathrm{Min}$. in ein Wasserbad von $60^{\circ}$ stellen, abkühlen, danach 20 Min. bei Raumtemperatur stehen lassen und zwischen 540 und $580 \mathrm{~nm}$ gegen den Reagenzienleerwert ablesen. Die Farbe ist bis zu einer Stunde stabil.

Berechnung:

$$
\frac{\text { Analyse-Analysenleerwert }}{\text { Standard-Analyse }} \cdot 164=\mathrm{mg} / 1 \mathrm{Hydroxyprolin}
$$

Um die Tagesausscheidung zu berechnen, wird das nach obenstehender Formel erhaltene Ergebnis mit der Urinausscheidung eines Tages (gemessen in Liter) multipliziert.

\section{Normalwerte}

Die von den verschiedenen Autoren angegebenen Normalwerte schwanken sehr stark. Bei der Vielzahl beschriebener Methoden ist das nicht verwunderlich. Es kommt hinzu, daß nicht alle in der Literatur, angegebenen Normalwerte sich auf Erwachsene und Heranwachsende getrennt beziehen. Bezieht man Jugendliche in die Normalwertberechnung mit ein, ergeben sich wesentlich höhere Werte wegen der erheblich erhöhten Hydroxyprolin-Ausscheidung während des Wachs- tums. GEYER (30) gibt einen 24-Stundenwert von $38,3 \pm 11,9 \mathrm{mg}$ für Männer und von $27 \pm 0,2 \mathrm{mg}$ für Frauen an. HARTMANN $(7,31)$ erwähnt $9-36 \mathrm{mg}$ als normale Tagesausscheidung. Ellis und GoldBerg (13), deren Methode wir mit einigen Änderungen übernommen haben, fanden bei 20 Männern und 20 Frauen folgende Werte für die 24Stunden-Ausscheidung nach 1 tägiger gelatinefreier Diät:

Männer $30,6 \pm 11,9(9,7-56,2) \mathrm{mg}$

Frauen $22,4 \pm 7,3 \quad(7,5-32,6) \mathrm{mg}$.

ZORAB (8) fand bei 44 14jährigen Knaben einenMittelwert von $110 \mathrm{mg} / \mathrm{Tag}$ und bei 53 12jährigen Mädchen $61,1 \mathrm{mg} /$ Tag.

In einer Untersuchung über die Aktivitätsdiagnostik der primär-chronischen Polyarthritis gehen HarTmanN und Mitarbeiter (31) von einer Hydroxyprolin-Ausscheidung von über $60 \mathrm{mg} / \mathrm{Tag}$ als Zeichen eines aktiv entzündlichen Prozesses aus. Unsere eigene NormalwertBestimmung bei 33 Frauen und 11 Männern ohne besondere Diät nach der hier beschriebenen Methode brachte folgende Ergebnisse:

Frauen (Alter 20-61 Jahre):

$20,2 \pm 6,5(5,71-44,9) \mathrm{mg}$

Männer (Alter 21 -59 Jahre):

$22,3 \pm 11,1(7,8-44,9) \mathrm{mg}$

Männer und Frauen zusammen:

$20,75 \pm 7,72(5,71-44,9) \mathrm{mg}$.

Auffällig ist dabei der gegenüber anderen Untersuchern geringere Einfluß des Geschlechts auf die Normalwerte und die weitgehende Übereinstimmung mit den Ergebnissen von Ellis und Goldberg (13), die im Gegensatz zu unseren nach 1 tägiger gelatinefreier Diät gewonnen wurden.

\section{Literatur}

1. Langness U., Dtsch. med. Wschr. 95, 2531 (1970). - 2. Rosimel K., L. Fourie, W. Böhm, W. E. Adam und G. Benecke, Med. Klin. 63, 410 (1968). - 3. Prockop, D. J. und K. I. Krvirikko, Annals Int. Med. 66, 1243 (1967). - 4. KeIser, A. R. und A. Sjoerdsma, J. Clin.Invest. 41, 1371 (1962). - 5. Keiser, H. R., J. R. Girl jr., A. SJOerdSMA und F. C. BartTER, J. Clin. Invest. 43,1,073 (1964). - 6. Cerda, J. J., P. P. Toskes, N. A. Shopa, J. H. Wilkinson, Clin. Chim. Acta, Amsterdam 27, 437 (1970). - 7. HartmanN, F., Klin. Wschr. 44, 1053 (1966). 8. Zorab, Lancet, London 1969/II, 1164. - 9. MeILMan, E., M. M. URIVETZKY und C. M. Rapoport, J. Clin. Invest. 42, 40 (1963). - 10. Krane, S. M., A. J. MuÑoz und E. D. Harris jr., J. Clin. Invest. 49, 716 (1970). - 11. Bergman, J. und R. LoxLEX, Analytic Chem. 35, 1961 (1963). - 12. BergmaN, J. und R. Loxley, Clin. Chim. Acta, Amsterdam 27, 347 (1970). - 13. Ellis und Goldberg, 7. Int. Kongr. klin. Chem., Genf 1969, Band 1, Meth. in Clin. Chem. (Karger Basel 1970). - 14. FrRSChern, H. E. und J. P. SHrll, Analytic Biochem. 14, 196 (1966). 15. Kivirikko, K. J., O. Laitinen und D. J. Prockop, Analytic Biochem. 19, 249 (1967). - 16. MöhleR, K. und N. ANTONACOpoulos, Zschr. Lebensmittel-Unters. u. -Forschung 106, 425
(1957). - 17. Stegemann, H. und K. Stalder, Clin. Chim. Acta, Amsterdam 18, 267 (1967). - 18. Le Roy, E. C., A. KapLAN, S. Udenfriend, A. Sjoerdsma, J. biol. Chemistry 239, 3350 (1964). - 19. MöhleR, K. und W. Vollex, Zschr. LebensmittelUnters. u. -Forschung 133, 158 (1967). - 20. PROCKOP, D. J. und S. UDENFriend, Analytic Biochem. 1, 228 (1960). - 21. StegemanN, H., Hoppe-Segler's Z. physiol. Chem. 411, 41 (1958). 22. Neuman, R. E. und M. A. Logan, J. biol. Chemistry 184 , 299 (1950). - 23. Bergman, J. und R. Loxley, Analyst. 94, 575 (1969). - 24. Rosano, C. L. in H. U. Bergmeyer, Methoden der enzymatischen Analyse, 2. Auf. Weinheim (1970). - 25. Koevoet, A. L. und J. D. BaArs, Clin. Chim. Acta, Amsterdam 25, 39 (1969). - 26. Stalder, K., Zschr. Analyt. Chem. 212, 196 (1965). - 27. Duponr, A., Clin. Chim. Acta, Amsterdam 18, 59 (1967). - 28. ARNETH, W. und R. HAMM, Zschr. LebensmittelUnters. 144, 301 (1970). - 29. Woessner, J. F. jr., Arch. Biochem. Biophysics 93, 440 (1961). - 30. Deutsch, E. und G. GeYer, Laboratoriumsdiagnostik, Verlag August Steinkopf, Berlin (1969). - 31. HartmanN, F., J. Rohde und A. Schmidr, Zschr. Rheumaforsch. 28, 263 (1969).
Dr. rer. nat. Heinz Haury 8 München 13

Schleißheimer Str. 343 


\section{W Walter de Gruyter Berlin-New York}

\section{Magenoperation und Magenoperierter}

Herausgegeben von

HEINRICH BARTELHEIMER, HANS. JOACHIM MAURER, HANS W. SCHREIBER unter Mitwirkung von Kurt Müller-Wieland. Mit 210 Abbildungen und 2 mehrfarbigen Tafeln. Groß-Oktav. XVI, 489 Seiten. 1969.

Ganzleinen DM 88, -

ISBN $311000819 X$

Weitere Mitarbeiter: Volker Becker -

Hans Berndt - Kurt Diwok -

Eduard Farthmann - Hellmuth

Freyberger - Max Gülzow - Alfred

Gütgemann - Werner Koch - Klaus

Krentz - Friedrich Kuhlencordt -

Otto Lindenschmidt - Adolf

Luchmann - Fritz Meissner - Walther

Pribilla - Friedrich Stelzner -

Karl-Otto Vorlaender - Egmont

Wildhirt - Ludwig Zukschwerdt

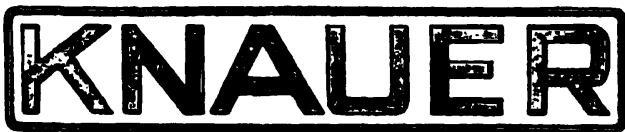

\section{ELEKTRON ISCHES}

\section{HALBMIKRO-OSMOMETER}

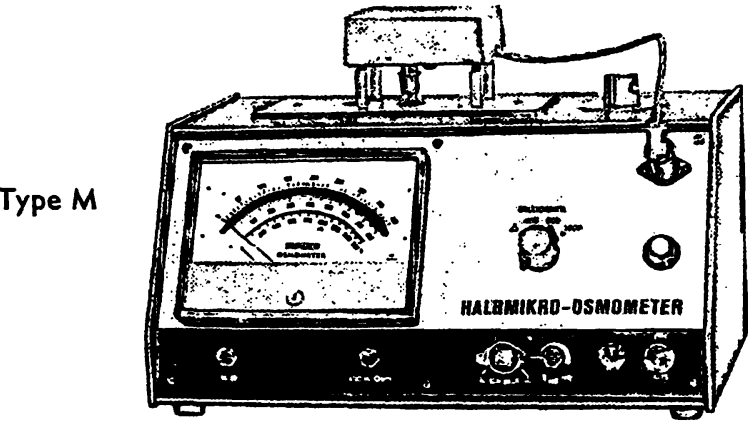

zur direkten Bestimmung der Osmolalität aller Körperflüssigkeiten wie Blut, Serum, Urin, Liquor durch Gefrierpunktmessung.

- Probevolumen nur $0,15 \mathrm{ml}$ oder $0,05 \mathrm{ml}$

- Betriebsbereitschaft sofort nach dem Einschalten

- Daver einer Messung ca. 2 Minuten

- Meßgenauigkeit 1-2 Milliosmol/kg bzw. 1\%

- Preis DM 3600, - + MWSt

- Lieferung ab Lager oder laufenden Serien

Weitere Spezialität: Komplettes System zur Molekulargewichtsbestimmung zwischen 100 und 1000000 durch Kryoskopie, Dampfdruck-Osmometrie und Membran-Osmometrie.

KG Dr.-Ing. Herbert Knaver \& Co. GmbH, 1 Berlin 37, Holstweg 18, Tel. 848705

Wir stellen aus: Leipziger Frühjahrsmesse, Halle 15, Stand 106

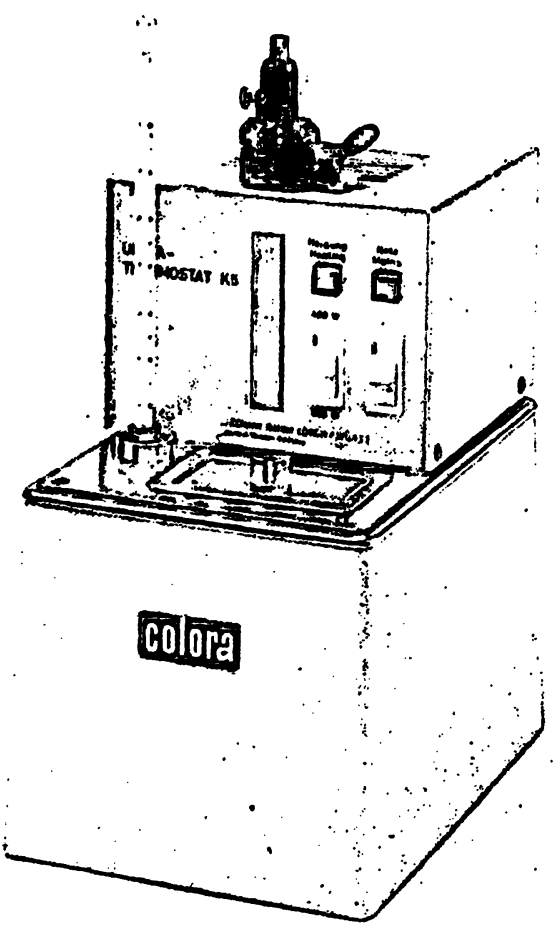

ColoraUltra-Thermostat $\mathrm{K} 5$

Kleinthermostat modernster Konstruktion für Temperierungen im Bad und im Umwälzverfahren. $\mathrm{K} 5 \mathrm{mit}$ Druckpumpe, K 5 DS mit Druck- und Saugpumpe. Lagerfreie Umwälzpumpe hoher Leistung. Funkentstörtes Elektronikrelais. Temperaturkon$\operatorname{stanz} \pm 0,02^{\circ} \mathrm{C}$. Hochwertiges Material in erstklassiger Verarbeitung. MetallteiledesTemperiersystemsaus rostfreiem Edelstahl. Ein zuverlässiges Gerät zu günstigem Preis.

\section{Colora Messtechnik GmbH}

\section{Lorch/Württ., Postfach 5} T (07172) 6041, FS 07-248886

Technische Büros (Verkauf und Kundendienst): 1000 Berlin 30, Kurfürstenstraße 84, T 2615200 2000 Hamburg 19, Osterstraße 63, T 4911034 , FS 02-12 947 3000 Hannover, An der Tiefenriede 45, T 884500 4000 Düsseldorf, Kronprinzenstr. 62, T 3201 64, FS 08-587 253 6000 Frankfurt a.M., Röderbergweg 4-6,T446031, FS04-11 216 8000 München 19, Dachauer Straße 175, T 193858

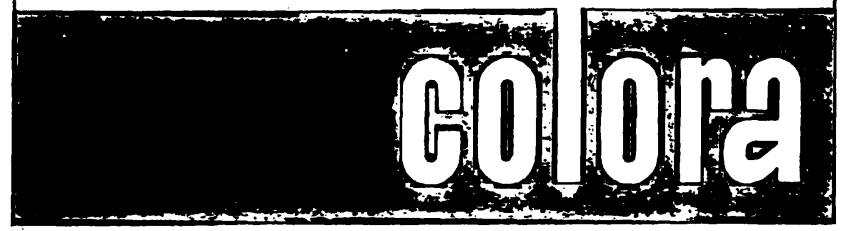




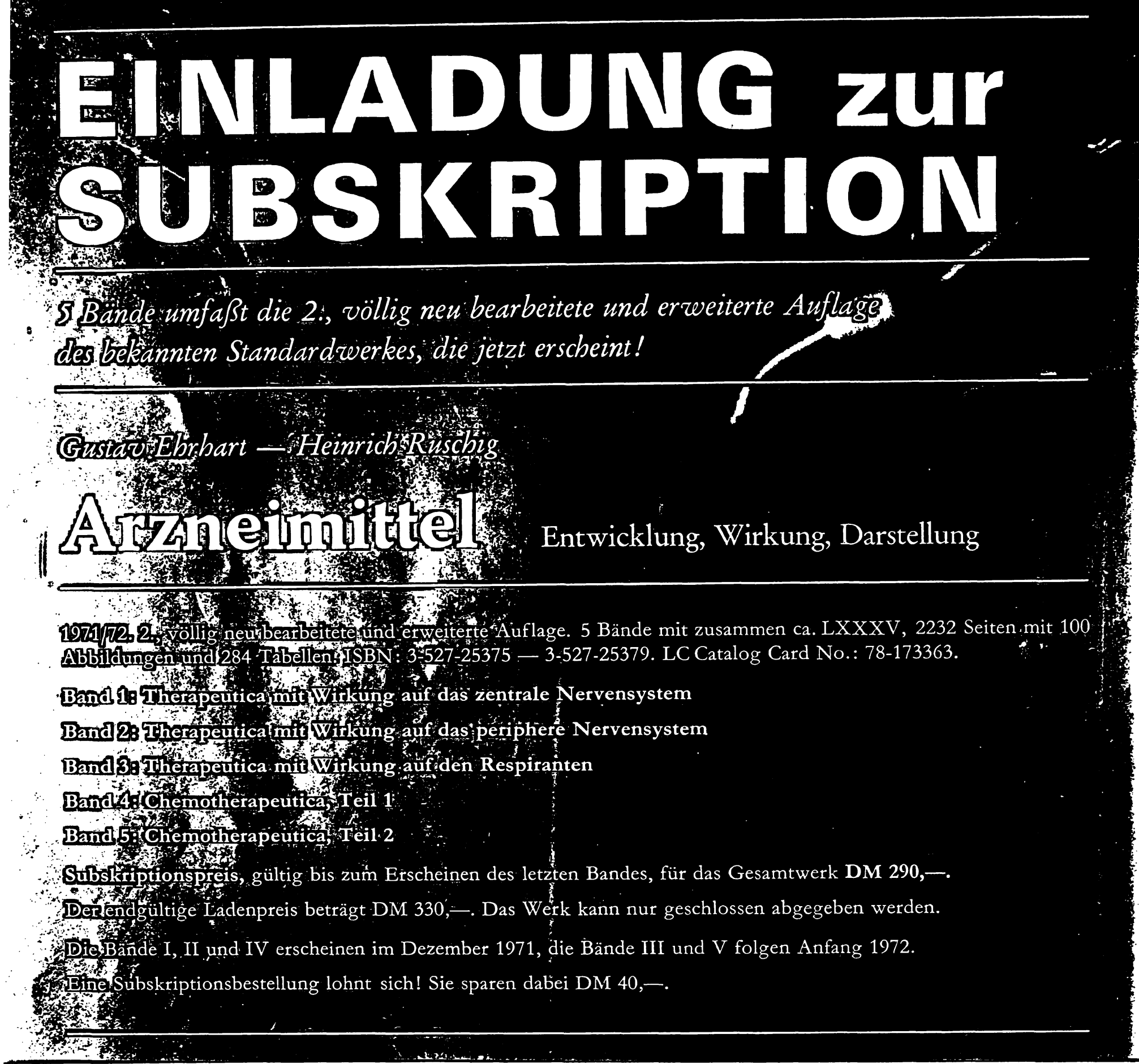

Die 2. Auflage dieser umfassenden Gesamtschau ist auf den neuesten Stand gebracht und entsprechend erweitert worden. Die Aufteilung in 5 Bände dient der besseren Handlichkeit. Das Werk ist ein unentbehrlicher Wegweiser für die medizinische Grundlagenforschung und Arzneimittelsynthese. Dieses große Úbersichtswerk in deutscher Sprache setzt Maßstäbe. Es bringt die Arzneimittel nach pharmakologischen Wirkungsbereichen sinnvoll gegliedert dar. Das didaktisch übersichtliche und prägnant geschriebene Kompendium bietet also wesentlich mehr, als nur tabellarische oder alphabetische Aufstellungen üblicher Handelsnamen und Mischpräparate.

Ausgeprägte dynamische Entwicklungsgebiete der Arzneimittelforschung, die bei Erscheinen der 1. Auflage noch nicht berücksichtigt werden konnten, sind in der fünfbändigen 2. Auflage ausführlich behandelt. Die 2. Auflage ist nach neuen Gesichtspunkten geordnet und enthält entsprechend zusätzliche Stichworte.

"Das Buch wird' Weltruf erlangen" urteilte die „Deutsche Medizinische
Wochenschrift" schon über die 1. Auflage. Überzeugen Sie sich von der Qualität und Nützlichkeit dieses unentbehrlichen Standardwerkes.<smiles>C1CC2CC1C2</smiles>

Verlag Chemie GmbH 694 Weinheim/Bergstr. Pappelallee 3 